\title{
A spectral nudging method for the ACCESS1.3 atmospheric model
}

\author{
P. Uhe ${ }^{1,2}$ and M. Thatcher ${ }^{1}$ \\ ${ }^{1}$ CSIRO Oceans and Atmosphere Flagship, 107-121 Station St, Aspendale, VIC 3195, Australia \\ ${ }^{2}$ Environmental Change Institute, University of Oxford, Oxford, UK \\ Correspondence to: P. Uhe (peter.uhe@ouce.ox.ac.uk)
}

Received: 7 August 2014 - Published in Geosci. Model Dev. Discuss.: 8 October 2014

Revised: 5 May 2015 - Accepted: 11 May 2015 - Published: 3 June 2015

\begin{abstract}
A convolution-based method of spectral nudging of atmospheric fields is developed in the Australian Community Climate and Earth Systems Simulator (ACCESS) version 1.3 which uses the UK Met Office Unified Model version 7.3 as its atmospheric component. The use of convolutions allow for flexibility in application to different atmospheric grids. An approximation using one-dimensional convolutions is applied, improving the time taken by the nudging scheme by 10-30 times compared with a version using a twodimensional convolution, without measurably degrading its performance. Care needs to be taken in the order of the convolutions and the frequency of nudging to obtain the best outcome. The spectral nudging scheme is benchmarked against a Newtonian relaxation method, nudging winds and air temperature towards ERA-Interim reanalyses. We find that the convolution approach can produce results that are competitive with Newtonian relaxation in both the effectiveness and efficiency of the scheme, while giving the added flexibility of choosing which length scales to nudge.
\end{abstract}

\section{Introduction}

Atmospheric modelling is a discipline that has impacts in many fields of scientific study as well as everyday life. For example, numerical weather prediction (Davies et al., 2005; Puri et al., 2013) provides us our daily weather forecasts and simulations of global climate (Taylor et al., 2012) give us forewarning of possible impacts of climate change. Global climate models are powerful tools, but they have limitations due to grid resolution, approximations to atmospheric physical processes (e.g. convection and turbulent mixing), and also because of incomplete or imperfect data sets such as for representing land use. Furthermore, since the atmosphere is a chaotic system, the simulated synoptic patterns deviate from observations over time. This makes it more difficult to evaluate modelled behaviour, since the advection of tracers depends on the synoptic-scale atmospheric circulation. In some cases, to reduce biases caused by these issues, it is useful to introduce a correction to align the model more closely with a host model, often an observational product such as the ERA-Interim reanalysis (ERAI; Dee et al., 2011). The process of adjusting dynamical variables of a model towards a host model is commonly known as nudging (Kida et al., 1991; Telford et al., 2008).

Nudging is useful for model development and scientific studies, where a more realistic atmospheric circulation can help determine errors or feedbacks in particular components of the model. Nudging in atmospheric models has been used to reduce the size of transport errors of trace gases for atmospheric chemistry (Telford et al., 2008) and carbon cycle modelling (Koffi et al., 2012), dynamically downscaling to finer resolution (Wang et al., 2004), and generating regional analyses (von Storch et al., 2000). Two popular approaches to nudging in atmospheric models are Newtonian relaxation (Telford et al., 2008) and spectral nudging (Waldron et al., 1996).

This paper describes an efficient method for implementing a convolution-based spectral nudging scheme in atmospheric models, which is demonstrated using the Australian Community Climate and Earth System Simulator (ACCESS; Bi et al., 2013; Dix et al., 2013). The spectral nudging scheme can support irregular grids, making the approach applicable to a wide range of other atmospheric models. We have also significantly improved its computational efficiency by approximating the spectral nudging using one-dimensional (1-D) convolutions, and show that this does not degrade the performance. A convolution approach for spectral nudging 
using a cubic grid has previously been described by Thatcher and McGregor (2009). However, this paper differs from the previous work, as the scheme in ACCESS has been designed to exploit the symmetries of the ACCESS latitude-longitude grid. This paper also provides an extended analysis to compare the performance of various configurations of nudging using Newtonian relaxation and spectral nudging.

ACCESS is a numerical model designed to simulate Earth's weather and climate systems. ACCESS is used for a wide range of applications from climate change scenarios and numerical weather prediction, to targeted scientific studies into areas such as atmospheric chemistry and aerosols, and the carbon cycle. ACCESS is composed of a number of different submodels, of which the atmospheric component is the UK Met Office Unified Model (UM; Davies et al., 2005; The HadGEM2 Development Team, 2011). The version of ACCESS used in this study, ACCESS1.3, includes the Community Atmosphere Biosphere Land Exchange model (CABLE; Kowalczyk et al., 2013) to represent the land surface. ACCESS often includes ocean and sea-ice components, but these components are not used in this study. A full description of ACCESS can be obtained from Bi et al. (2013).

Nudging was originally implemented in the UM at the University of Cambridge, UK (Telford et al., 2008), using a Newtonian relaxation method. This applies a correction to the model at every time step, calculated from the difference between the host model and the UM. The fields that are nudged are the key dynamical variables; $\Theta$ (potential temperature), $U$ (zonal wind) and $V$ (meridional wind).

An alternate approach to Newtonian relaxation is spectral nudging (von Storch et al., 2000; Thatcher and McGregor, 2009; Waldron et al., 1996). The spectral nudging scheme builds upon and expands the already existing Newtonian relaxation nudging code in the UM. It applies a low-pass spectral filter on the correction calculated as for the relaxation nudging, so the correction is only applied to large spatial scales. The spectral filter is applied using a convolution with a two-dimensional (2-D) Gaussian function. A convolutionbased filter was chosen rather than using a more conventional discrete Fourier transform, as it is simple to implement a parallel version within the UM framework and has the potential to be generalized to irregular and limited area grids. It also operates on the physical distance between grid points, which makes it straight forward to apply consistently across the whole globe and does not require special treatment of the poles. Spectral nudging gives the flexibility of being able to nudge the large-scale features of the model towards the host, while allowing the small scales to be determined by the model's own physics. Because of this, spectral nudging is particularly useful in regional climate modelling (Denis et al., 2002; Kanamaru and Kanamitsu, 2007; Kida et al., 1991) and dynamical downscaling (Liu et al., 2012). In these cases, the model resolution is finer than the host model, so there is no information to nudge the finest length scales of the model towards, preventing the effective use of relaxation nudging.

The paper is structured as follows: Sect. 2 covers the implementation and configuration of nudging in ACCESS. This includes Sect. 2.1 covering relaxation nudging, then Sect. 2.2 describing the implementation of the spectral filter and the convolution method used to implement it. A 1-D filter that approximates the 2-D filter is described in Sect. 2.3. The 1-D filter gives significant improvements in the speed of calculating the filter and reduces the amount of message passing. The set-up of the model used for simulations presented in this document is covered in Sect. 2.4.

The performance of the spectral nudging is analysed in Sect. 3. This is split up into subsections relating to different indicators of its performance or looking at the behaviour from different parameter choices. Section $3.1 \mathrm{com}-$ pares nudged variables of the ACCESS model with ERAI, as well as the unconstrained fields of mean sea level pressure (MSLP) and precipitation. Section 3.2 compares the performance of the 1-D and 2-D spectral filters. Section 3.3 compares a number of different nudging configurations to see how closely they converge towards ERAI, and the effect of varying the spectral filter length scale. Lastly, Sect. 3.4 investigates the effect of varying the period of nudging, comparing its effect on the temporal spectrum and run times.

\section{Nudging implementation}

The process of nudging aims at perturbing prognostic variables $\psi_{\mathrm{m}}$ of a model (e.g. ACCESS) toward the corresponding variable $\psi_{\mathrm{h}}$ of a host model (e.g. ERAI). The following section relates how nudging is implemented for each of the different methods used in this paper.

\subsection{Newtonian relaxation}

The standard Newtonian relaxation is applied by taking the difference between $\psi_{\mathrm{m}}$ and $\psi_{\mathrm{h}}, \Delta \psi=\psi_{\mathrm{m}}-\psi_{\mathrm{h}}$, and using this to correct the model,

$\psi_{\mathrm{m}} \rightarrow \psi_{\mathrm{m}}-\alpha \Delta \psi$

Here $\alpha \in[0,1]$ is a dimensionless constant determining the strength of nudging. $\alpha$ is related to the concept of an $e$ folding time, which is the length of time to reduce the error by $e^{-1}$, where $\alpha<1$. The $e$-folding time is $\Delta t / \alpha$ where $\Delta t$ is the period of nudging. For example, a $6 \mathrm{~h} e$-folding time with nudging applied every half hour corresponds to $\alpha=1 / 12$. $\alpha$ has vertical dependence, and is set to 0 below $1000 \mathrm{~m}$ (i.e. the typical planetary boundary layer height). This helps in avoiding conflict between the nudging and the atmospheric model, since the behaviour of the atmosphere in the boundary layer is strongly influenced by the land surface, which can be different between the model and its host. $\alpha$ is also typically ramped down linearly from its full strength to zero 
over several model levels to reduce the discontinuity between the nudged and non-nudged regions. It can also be ramped down at the top of the atmosphere to avoid any conflict that may occur due to top boundary conditions of the model.

The code used for the relaxation nudging is based on code from Telford et al. (2008) with some modifications. The code was restructured to improve parallelism when spatially interpolating host data and to use the ERAI data set as the host model instead of other reanalysis products.

\subsection{Spectral nudging}

Spectral nudging extends the Newtonian relaxation method by taking the correction term and applying a spectral (lowpass) filter so that large spatial wavelengths are adjusted while smaller wavelengths are left essentially unperturbed. The method chosen to do this is based on Thatcher and McGregor (2009), using a convolution of $\Delta \psi$ with a Gaussian function, $w$, to implement the filter. However, the approach in this paper differs from previous work in its application to the ACCESS grid, requiring different implementation of the convolution for different underlying grids.

The correction for spectral nudging is applied as follows:

$\psi_{\mathrm{m}} \rightarrow \psi_{\mathrm{m}}-\alpha(\Delta \psi * w)$,

where $*$ is the convolution operator. The convolution is calculated on the surface of a sphere (assumed to have radius $R=1$ ). This results in

$\Delta \psi * w=\iint \Delta \psi\left(\theta^{\prime}, \phi^{\prime}\right) w\left(\theta^{\prime}-\theta, \phi^{\prime}-\phi\right) \cos \left(\phi^{\prime}\right) \mathrm{d} \phi^{\prime} \mathrm{d} \theta^{\prime}$

where the Gaussian weighting function is

$w\left(\theta^{\prime}-\theta, \phi^{\prime}-\phi\right)=\frac{1}{b} \exp \left(\frac{-\Delta \sigma^{2}}{2 \lambda^{2}}\right)$.

$\lambda$ is the standard deviation of the Gaussian function, which is referred to as the nudging length scale. $\theta$ and $\phi$ are the azimuthal angle and polar angle, respectively, and $\theta^{\prime}$ and $\phi^{\prime}$ are dummy co-ordinates that are integrated over. $b$ is a normalization factor, $b=\iint \exp \left(\frac{-\Delta \sigma^{2}}{2 \lambda^{2}}\right) \mathrm{d} \phi^{\prime} \mathrm{d} \theta^{\prime}$. Note that $b$ is evaluated after the expression is discretized. $\Delta \sigma\left(\theta^{\prime}-\theta, \phi^{\prime}-\right.$ $\phi)$ is the distance of a chord between the two points, $\left(\theta^{\prime}, \phi^{\prime}\right)$ and $(\theta, \phi)$ :

$\Delta \sigma=2 \arcsin \left(\frac{C}{2}\right)$

where $C\left(\theta^{\prime}-\theta, \phi^{\prime}-\phi\right)$ is the Cartesian distance between the points $\left(\theta^{\prime}, \phi^{\prime}\right)$ and $(\theta, \phi)$. Combining and discretizing Eqs. (3), (4) and (5), we get the correction that is applied by the scale-selective filter.

The ACCESS grid is horizontally decomposed into domains that are assigned to individual processors. The calculation of the convolution at any point requires a global sum.
Global information is not stored on individual processors, so the message passing interface (MPI) is used to gather the $\Delta \psi$ arrays handled by each processor into a global array, and broadcast them to all processors. Each processor calculates the convolution just for its domain using this global information.

The naive implementation of the spectral filter involves a large computational effort (of order $N^{2}$ computations for $N$ horizontal grid points). A spectral filter could be implemented more efficiently via a fast Fourier transform (FFT), or a spherical harmonic transform, requiring order $N \log _{2} N$ computations, but the convolution gives much greater flexibility to be used with different grid configurations, from the regular latitude-longitude grid to irregular or limited area grids. To mitigate the computational effort of the convolution, a 1-D approximation to the convolution has been developed, described in the following section.

\subsection{1-D filter}

To improve the computational efficiency of the spectral nudging scheme, the 2-D convolution can be separated into two 1-D convolutions, thereby reducing the computational effort to order $N^{3 / 2}$. The 2-D convolution is separated by splitting the Gaussian function into parts that depend solely on latitude or longitude. The two integrals in the 2-D filter can then be evaluated separately as two 1-D convolutions. The expression for the two 1-D convolutions is equal to the 2-D convolution on a flat Cartesian grid, but is an approximation on a curved surface such as the global latitude-longitude grid.

$$
\begin{aligned}
w\left(\theta^{\prime}-\theta, \phi^{\prime}-\phi\right) & \approx \frac{1}{b} w\left(\theta^{\prime}-\theta, \phi\right) w\left(\theta^{\prime}, \phi^{\prime}-\phi\right) \\
& \approx \frac{1}{b} \exp \left(\frac{-\Delta \sigma\left(\theta^{\prime}-\theta, \phi\right)^{2}}{2 \lambda^{2}}\right) \\
& \exp \left(\frac{-\Delta \sigma\left(\theta^{\prime}, \phi^{\prime}-\phi\right)^{2}}{2 \lambda^{2}}\right) .
\end{aligned}
$$

A 1-D convolution is applied in one direction, then another 1$\mathrm{D}$ convolution is applied on the result of the first convolution.

$$
\begin{aligned}
\Delta \psi * w & \approx \frac{1}{b}\left[\Delta \psi * w\left(\theta^{\prime}, \phi^{\prime}-\phi\right)\right] * w\left(\theta^{\prime}-\theta, \phi\right) \\
& \approx \frac{1}{b} \int\left[\int w\left(\theta^{\prime}, \phi^{\prime}-\phi\right) \Delta \psi\left(\theta^{\prime}, \phi^{\prime}\right) \cos \left(\phi^{\prime}\right) \mathrm{d} \phi^{\prime}\right] \\
& \cdot w\left(\theta^{\prime}-\theta, \phi\right) \mathrm{d} \theta^{\prime} .
\end{aligned}
$$

Since the integrals are computed independently, $w$ is calculated along horizontal rows and columns separately, not over the whole globe. Consequently, the code scales better with increasing numbers of processors. As well as computational speedup, this reduces communication bottlenecks from data passed through MPI. Rather than global arrays being broadcast to every processor, each processor only needs 
data passed from processors associated with the same rows or columns of the horizontal grid.

Using this 1-D approximation, there is a choice in which convolution to apply first (i.e. either the zonal or meridional directions). Swapping the order of the integrals (convolutions) results in numerically different solutions. It is found that to reduce the error it is best to apply the convolution first along the latitudinal direction, then longitudinally. This is discussed in Sect. 3.2, which compares the different orderings of the 1-D filter with the 2-D filter.

It also needs to be noted that the 1-D spectral filter is dependent on the model grid and the way the grid is decomposed into domains for each processor. The configuration of the ACCESS grid allows for the convolution to be computed along rows of equal latitude or longitude and for those results to be efficiently distributed to rows or columns of processors. This approach needs to be modified for grids which do not have these symmetries. See Thatcher and McGregor (2009) for an example of a 1-D spectral filter applied on a cubic grid.

\subsection{Model configuration/description}

This paper uses simulations of ACCESS, in the ACCESS1.3 atmosphere-only configuration (Bi et al., 2013). This uses the atmospheric model UM vn7.3, CABLE 1.8 (Kowalczyk et al., 2013), as well as prescribed sea-surface temperatures and sea-ice concentrations. The model horizontally uses a N96 grid (uniform latitude longitude grid with $1.875^{\circ}$ east-west and $1.25^{\circ}$ north-south resolution). It has 38 vertical levels which are terrain following hybrid height levels, representing heights from $10 \mathrm{~m}$ to $36 \mathrm{~km}$. The model was run with a 30 min time step.

A series of 1-year simulations were run, starting from 1 January 1990, each initialized in the same state, from a previous climate simulation, i.e. with an initial state unrelated to any historical synoptic patterns. The only differences between simulations were in the nudging configuration. These short experiments were chosen to evaluate the performance of different nudging methods and choice of nudging parameters. Longer climate simulations may also provide more in depth insight into biases in the nudging scheme, but this evaluation is beyond the scope of this paper.

The nudging component used the ERAI reanalysis product as the host model, provided at 6 hourly intervals. The ERAI data was linearly interpolated temporally to each time step. It was interpolated horizontally using bi-cubic interpolation, from its native $0.75^{\circ}$ east-west and $0.75^{\circ}$ north-south to a resolution of $1.875^{\circ}$ east-west by $1.25^{\circ}$ north-south, matching the grid used by the ACCESS1.3 atmosphere. This is a higher resolution than the ERA-40 reanalysis used in Telford et al. (2008) of $3.75^{\circ}$ east-west by $2.5^{\circ}$ north-south.

The ERAI data set was interpolated vertically to the ACCESS1.3 model levels, using the vertical interpolation developed in Telford et al. (2008), based on a piece-wise linear interpolation with respect to the natural logarithm of the air pressure. Some nudging methods include corrections to the vertical interpolation to account for the differences in orography between the simulation model and the host model (ACCESS1.3 and ERAI in this paper, respectively), such as exploiting the lapse rate to correct the interpolation of air temperature. However, since our goal in this manuscript is to evaluate the scale-selective filter compared to Newtonian relaxation, we have elected to retain the original interpolation scheme of Telford et al. (2008) for this study. Nevertheless, orographic adjustment for interpolated fields is an important topic that we intend to address in further work.

Nudging was applied to potential temperature $(\Theta)$, zonal wind $(U)$ and meridional wind $(V)$. The choice of which prognostic variables to nudge is an important aspect of the experiment design. Kanamaru and Kanamitsu (2007) argued that the $U$ and $V$ wind components, temperature, water vapour and surface pressure, all needed to be nudged to sufficiently constrain the large-scale biases of the model. Jeuken et al. (1996) also highlighted the effect nudging can have on the model physics if the nudging terms become too large. For simplicity, we have chosen to nudge the $\Theta, U$, and $V$ so as to be consistent with the nudging approach used by Telford et al. (2008). Note that we specifically avoid nudging the water vapour due to its potentially highly non-linear behaviour in the presence of clouds. We specifically consider whether the nudging is unbalancing the model in Sect. 3.4 in the context of the temperature temporal spectra.

The nudging adjustment was only applied from vertical level 7, corresponding to about $1 \mathrm{~km}$ in height above the surface terrain. The nudging amplitude $\alpha$ was ramped up from 0 to the full strength over three vertical levels so as to reduce the discontinuity between nudged and non-nudged parts of the atmosphere. $\alpha$ was also ramped down over the top three vertical levels of the model.

The parameters varied in the experiments were the nudging method, nudging period, maximum nudging strength, and spectral filter length. The relaxation nudging is always applied every time step, and the spectral filter can be applied at frequencies that are multiples of the time step and divide into $6 \mathrm{~h}$ (e.g. $0.5,1,2,3$ or $6 \mathrm{~h}$ ). Most spectral nudging simulations analysed in this manuscript have nudging applied at a frequency of $1 \mathrm{~h}$, which is justified in Sect. 3.4.

Simulations presented use a maximum nudging strength corresponding to either a $1 \mathrm{~h} e$-folding time (referred to as hard nudging) or $6 \mathrm{~h} e$-folding time (referred to as soft nudging). We note that soft nudging is used in Telford et al. (2008) and is a common choice for relaxation nudging. In this paper we find it useful to compare soft relaxation nudging with the soft spectral nudging and hard relaxation nudging with hard spectral nudging. Simulations were run with a range of filter length scales, from 0.03 to 0.5 radians. 

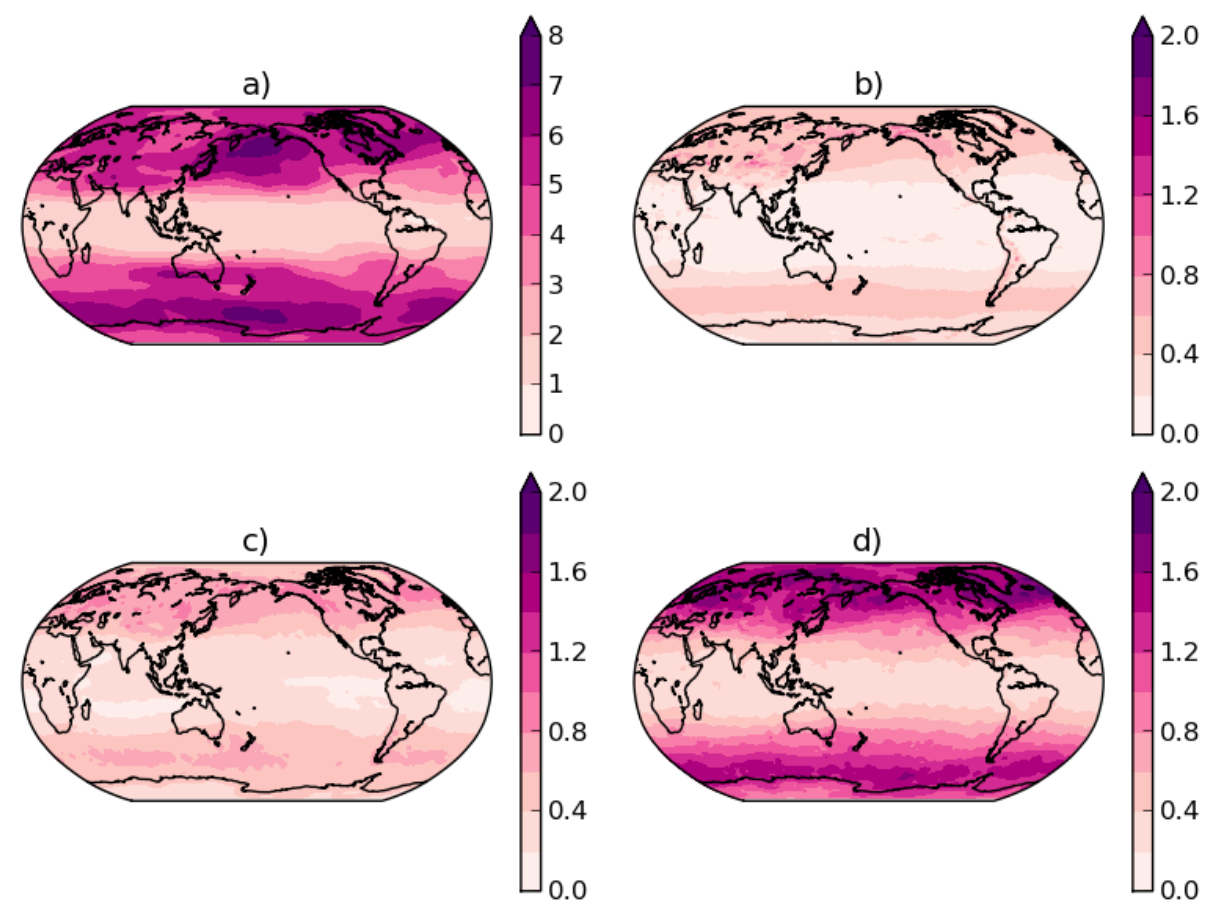

Figure 1. Spatial distributions of the RMSE in air temperature of ACCESS simulations. This is measured in Kelvin on a 2-D horizontal plane at $250 \mathrm{hPa}$ and averaged over 1-year simulations excepting the first 10 days. (a) is the control with no nudging. (b) is the relaxation nudging with hard nudging. (c and d) are spectral nudging using the 1-D filter with hard nudging, applied once an hour. Different nudging length scales were used: $\lambda=0.1$ in (c) and $\lambda=0.2$ in (d). Note, for clarity, (a) uses a different scale for the contours.

\section{Results and discussion}

To determine the performance of the spectral filter, we look at the nudged runs compared with ERAI, as well as comparing with a control simulation without nudging. The control simulation also gives an indication of the behaviour of the nudging tendencies that were required to change the evolution of the simulation. The analysis was conducted on the nudged air temperature and wind fields, measured on planes of constant pressure at 250,500 and $850 \mathrm{hPa}$. Note that although the potential temperature $\Theta$ is nudged, we actually evaluate the air temperature $T$ when comparing the simulated results with ERAI. Two unconstrained fields, MSLP and precipitation, were also evaluated. Except where specified otherwise, the whole 1-year simulations excepting the first 10 days was used. Excluding this period from the analysis ensures the atmosphere is settled fully into the nudged state.

After describing the impact of nudging in Sect. 3.1, we compare different implementations of the 1-D and 2-D spectral filters in Sect. 3.2 and then evaluate the influence of using different spectral filter parameters in Sect. 3.3. Lastly, Sect. 3.4 gives a justification for the selection of the period of application of spectral nudging which is used throughout this manuscript.

\subsection{Analysis of mean state and variance in the nudged model}

\subsubsection{Effect of nudging on nudged atmospheric fields}

Figure 1 shows the spatial distribution of the root mean squared error (RMSE) at $250 \mathrm{hPa}$ for different ACCESS simulations, where we are defining the error as the difference between ACCESS and ERAI. It is calculated over a 1-year simulation, excepting the first 10 days, for the 6 hourly intervals the ERAI data is provided on. For these plots, a control simulation with no nudging is compared against simulations using relaxation nudging and spectral nudging. The different behaviour between the nudged and control simulations provides an indication of the strength of the nudging tendencies. The 1-D filter was chosen as the preferred method of spectral nudging, as discussed further in Sect. 3.2. In all cases, the nudged runs have much smaller errors than the control simulation, indicating closer agreement with ERAI. The spectral filter with small length scales nudged (Fig. 1c) results in behaviour similar to the relaxation nudging (Fig. 1b). As the filter length scale is increased, larger wavelengths are able to deviate from ERAI, and the magnitudes of the deviations are larger (Fig. 1d).

Figures 2 and 3 show the same data as Fig. 1, except lower in the atmosphere, at levels of 500 and $850 \mathrm{hPa}$, respectively. As the height decreases, we notice differences in 

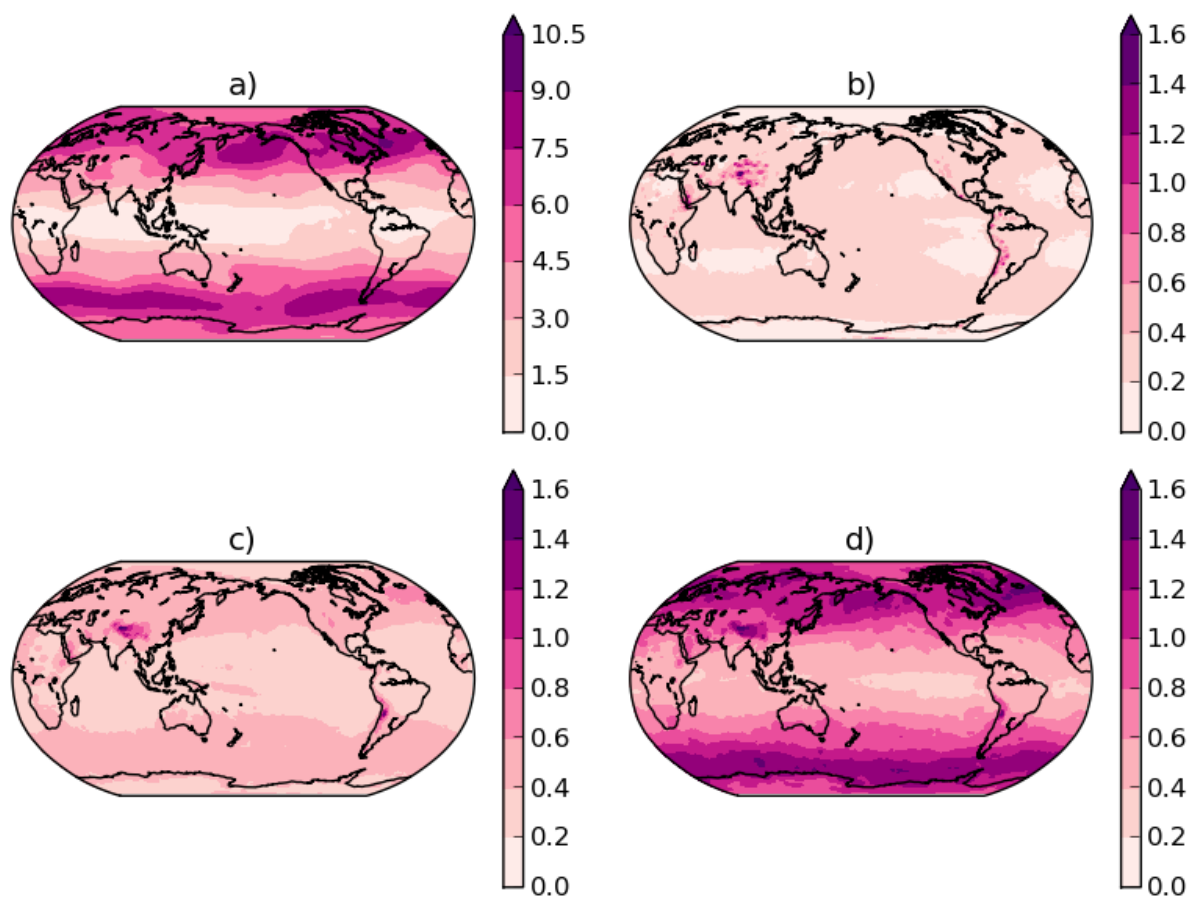

Figure 2. Spatial distributions of the RMSE in air temperature of ACCESS simulations. This is measured in Kelvin on a 2-D horizontal plane at $500 \mathrm{hPa}$ and averaged over 1-year simulations excepting the first 10 days. (a) is the control with no nudging. (b) is the relaxation nudging with hard nudging. (c and d) are spectral nudging using the 1-D filter with hard nudging, applied once an hour. Different nudging length scales were used: $\lambda=0.1$ in (c) and $\lambda=0.2$ in (d). Note, for clarity, (a) uses a different scale for the contours.
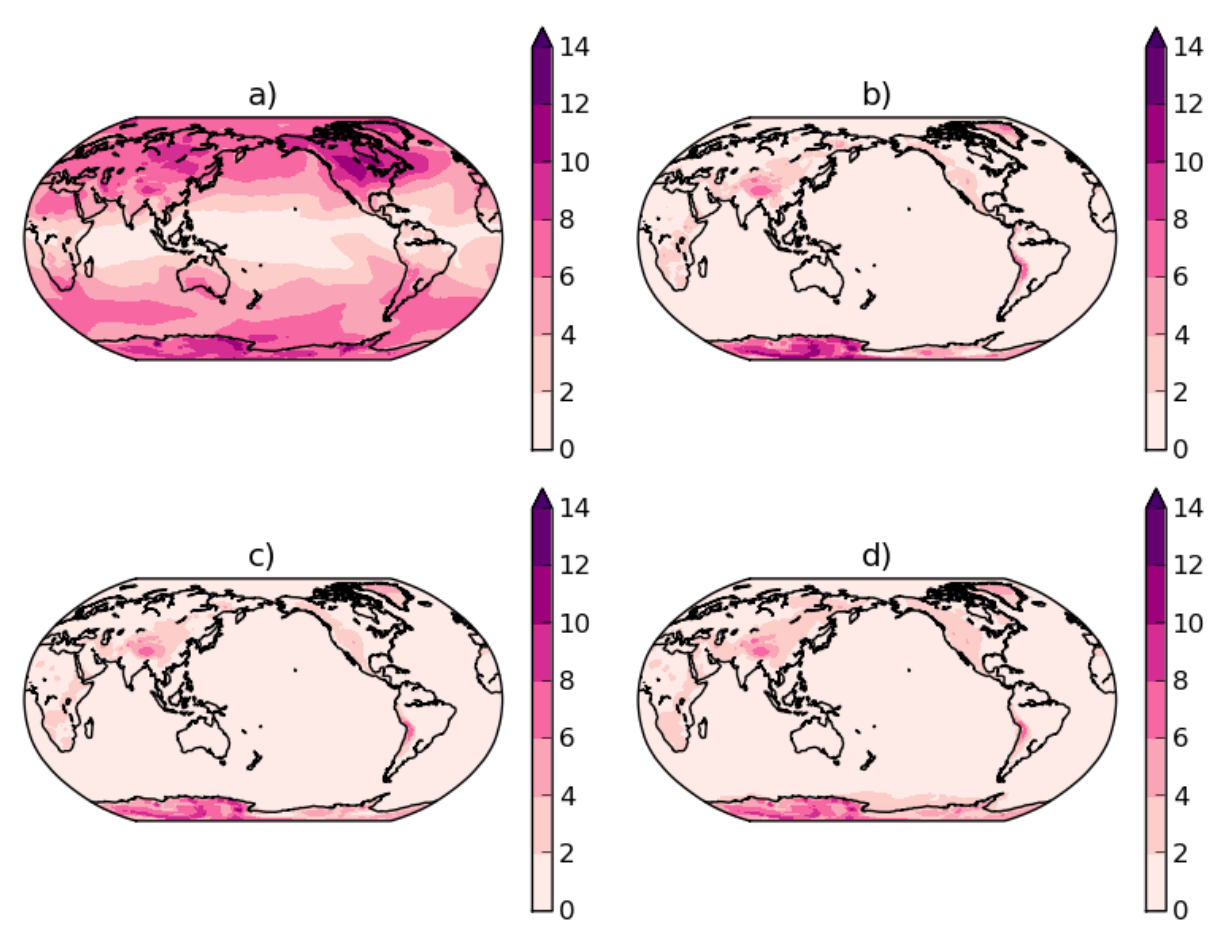

Figure 3. Spatial distributions of the RMSE in air temperature of ACCESS simulations. This is measured in Kelvin on a 2-D horizontal plane at $850 \mathrm{hPa}$ and averaged over 1-year simulations excepting the first 10 days. (a) is the control with no nudging. (b) is the relaxation nudging with hard nudging. (c and d) are spectral nudging using the 1-D filter with hard nudging, applied once an hour. Different nudging length scales were used: $\lambda=0.1$ in (c) and $\lambda=0.2$ in (d). 


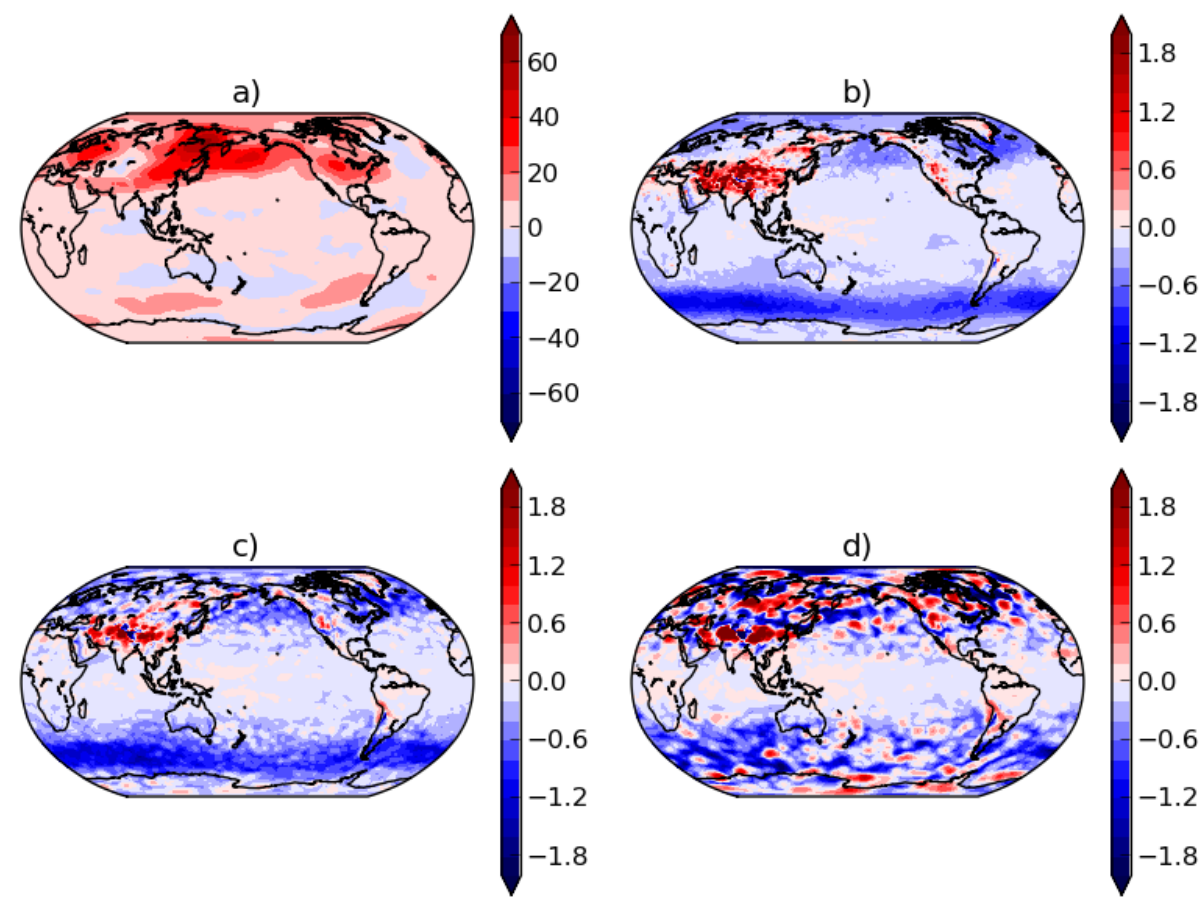

Figure 4. Spatial distributions of the difference in variance of air temperature between ACCESS simulations and ERAI. This is measured in Kelvin squared, on a 2-D horizontal plane at $500 \mathrm{hPa}$ and averaged over 1-year simulations excepting the first 10 days. (a) is the control with no nudging. (b) is the relaxation nudging hard nudging. (c and d) are spectral nudging using the 1-D filter with hard nudging, applied once an hour. Different nudging length scales were used: $\lambda=0.1$ in (c) and $\lambda=0.2$ in (d). Note, for clarity, (a) uses a different scale for the contours.

the simulation over high orographic features such as the Himalayas, Antarctica or the Andes. These differences become more pronounced with the $850 \mathrm{hPa}$ results, which is close to the lowest atmospheric levels that are nudged. We would expect there to be some differences between ACCESS and ERAI near the surface due to different representation of landsurface processes and different boundary layer parametrizations between the different atmospheric models. However, the largest errors are located where there is likely to be a mismatch in orographic height between ACCESS and ERAI and may suggest a limitation of the current method of interpolating ERAI to the ACCESS grid.

The $U$ and $V$ winds show similar trends in the relative RMSE between different simulations as those shown for temperature in Figs. 1, 2 and 3. This is demonstrated by the global average RMSE of these fields, shown in Tables 2 and 3 . These tables present data at atmospheric levels of 250,500 and $850 \mathrm{hPa}$. We note that all of the nudging simulations are more strongly constrained in RMSE than the control simulation with no nudging. This is true for each of the variables, at each level. At $850 \mathrm{hPa}$, which is close to the lowest atmospheric levels that are nudged, the $U$ and $V$ winds have an average RMSE similar to the higher atmospheric levels. In contrast, the air temperature average RMSE shown in Fig. 1 is multiple times greater than the higher atmospheric levels. This shows that temperature is more affected by the sur- face and orographic differences. We also note that the hard nudging simulations are more constrained in RMSE than the equivalent simulations with soft nudging in all cases.

Since we intend to use the nudging in the simulation over climate timescales (i.e. decades), it is useful to determine how well the simulation predicts the variance as well as the mean air temperature. Figure 4 shows the differences in the variance of air temperature between the ACCESS simulations and ERAI at $500 \mathrm{hPa}$. Note that ACCESS consistently overestimates the variance of the air temperature in the control experiment compared to ERAI (Fig. 4a), presumably as a consequence of imperfect physical parametrizations. We note that this overestimate of the variance in air temperature is reduced by the nudging, with the difference in variance for Fig. $4 \mathrm{~b}-\mathrm{d}$ being an order of magnitude less than for Fig. 4a.

It is also useful to compare the performance of the model between small and large spatial scales. The RMSE gives the error grid point by grid point, at the smallest length scale. To evaluate the error at the largest length scale (the whole globe), the global mean of the difference between ACCESS and ERAI can be used. We refer to this as the global average error (GAE). Values for the GAE are included in Tables 1,2 and 3 for $T, U$ and $V$, respectively. This covers each of the atmospheric fields nudged in our simulations at different heights in the atmosphere. In addition to the values in the tables, we note that the GAE tends to fluctuate rather 

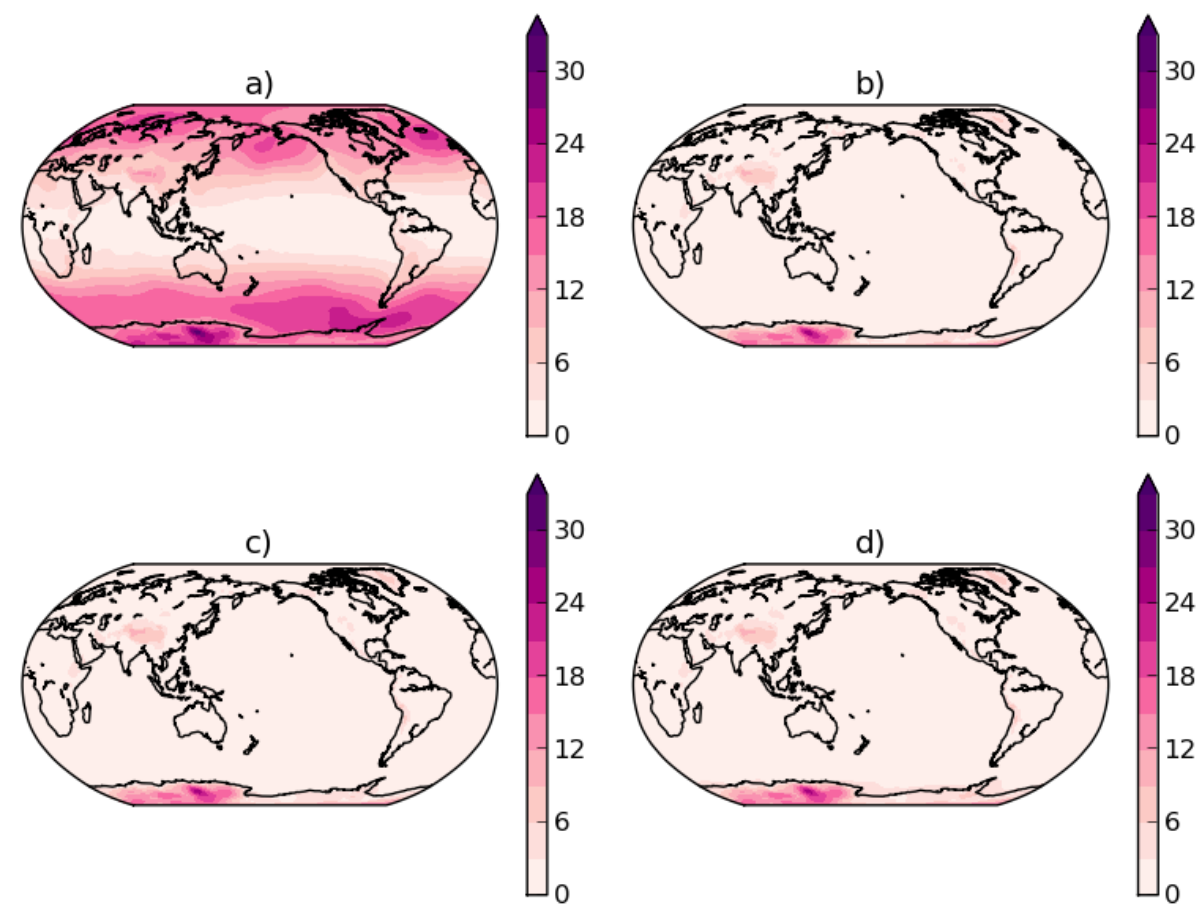

Figure 5. Spatial distributions of the RMSE for MSLP in hectopascal, between ACCESS simulations and ERAI. This is averaged over daily mean values for 1-year simulations excepting the first 10 days. (a) is the control with no nudging. (b) is the relaxation nudging hard nudging. (c and d) are spectral nudging using the 1-D filter with hard nudging, applied once an hour. Different nudging length scales were used: $\lambda=0.1$ in (c) and $\lambda=0.2$ in (d).
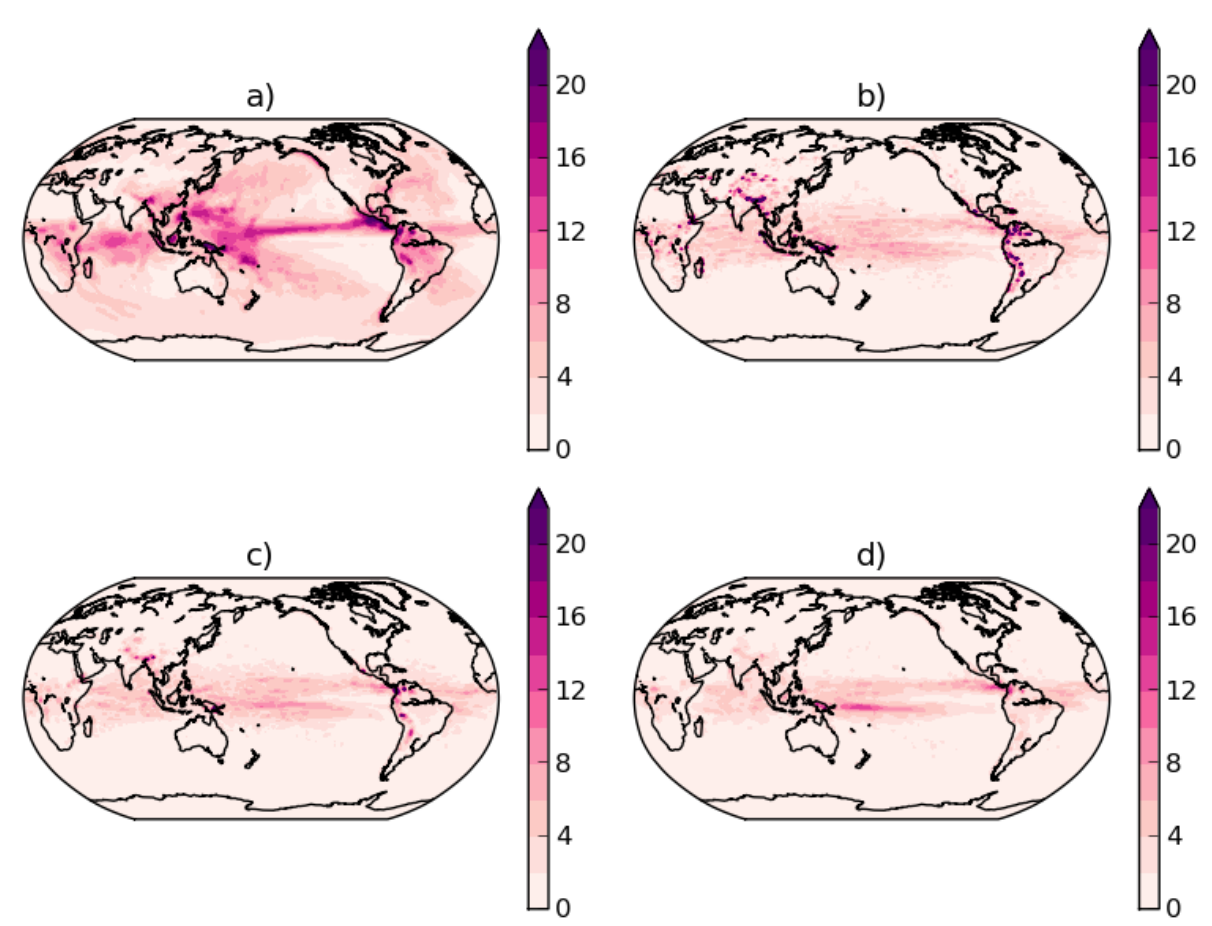

Figure 6. Spatial distributions of the RMSE of monthly mean precipitation in millimetres per day, between ACCESS simulations and ERAI. This is averaged over a 1-year simulation. (a) is the control with no nudging. (b) is the relaxation nudging hard nudging. (c and d) are spectral nudging using the 1-D filter with hard nudging, applied once an hour. Different nudging length scales were used: $\lambda=0.1$ in (c) and $\lambda=0.2$ in $(\mathbf{d})$. 
Table 1. Comparison of RMSE and GAE in air temperature measured in Kelvin, for 1-year simulations excepting the first 10 days, using different nudging methods. Spectral nudging experiments use nudging applied once an hour.

\begin{tabular}{lrrrrrrr}
\hline Experiment & \multicolumn{3}{c}{ RMSE } & & \multicolumn{3}{c}{ GAE } \\
\cline { 2 - 5 } \cline { 6 - 8 } & $250 \mathrm{hPa}$ & $500 \mathrm{hPa}$ & $850 \mathrm{hPa}$ & & $250 \mathrm{hPa}$ & $500 \mathrm{hPa}$ & $850 \mathrm{hPa}$ \\
\hline Control & 4.25 & 4.69 & 5.08 & & -0.37 & 0.13 & 0.39 \\
Relaxation, soft & 0.42 & 0.38 & 1.37 & & 0.030 & 0.033 & 0.27 \\
Relaxation, hard & 0.32 & 0.26 & 1.39 & & 0.15 & 0.073 & 0.39 \\
Spectral, soft, $\lambda=0.1$ & 0.68 & 0.64 & 1.55 & & -0.026 & -0.042 & 0.13 \\
Spectral, hard, $\lambda=0.03$ & 0.35 & 0.29 & 1.37 & & 0.13 & 0.057 & 0.37 \\
Spectral, hard, $\lambda=0.1$ & 0.45 & 0.41 & 1.36 & & 0.081 & -0.001 & 0.18 \\
Spectral, hard, $\lambda=0.2$ & 0.90 & 0.83 & 1.62 & & 0.063 & -0.021 & 0.11 \\
\hline
\end{tabular}

Table 2. Comparison of RMSE and GAE in $U$ measured in metres per second, for 1-year simulations excepting the first 10 days, using different nudging methods. Spectral nudging experiments use nudging applied once an hour.

\begin{tabular}{|c|c|c|c|c|c|c|}
\hline \multirow[t]{2}{*}{ Experiment } & \multicolumn{3}{|c|}{ RMSE } & \multicolumn{3}{|c|}{ GAE } \\
\hline & $250 \mathrm{hPa}$ & $500 \mathrm{hPa}$ & $850 \mathrm{hPa}$ & $250 \mathrm{hPa}$ & $500 \mathrm{hPa}$ & $850 \mathrm{hPa}$ \\
\hline Control & 18.26 & 3.09 & 8.25 & 0.70 & 0.21 & -0.42 \\
\hline Relaxation, soft & 1.32 & 1.00 & 1.35 & -0.044 & 0.012 & -0.12 \\
\hline Relaxation, hard & 0.77 & 0.58 & 0.97 & -0.029 & 0.015 & -0.068 \\
\hline Spectral, soft, $\lambda=0.1$ & 2.67 & 2.28 & 2.28 & -0.060 & 0.013 & -0.12 \\
\hline Spectral, hard, $\lambda=0.03$ & 1.01 & 0.78 & 1.12 & -0.027 & 0.014 & -0.068 \\
\hline Spectral, hard, $\lambda=0.1$ & 1.74 & 1.54 & 1.76 & -0.028 & 0.009 & -0.072 \\
\hline Spectral, hard, $\lambda=0.2$ & 3.59 & 3.09 & 2.71 & -0.030 & 0.008 & -0.072 \\
\hline
\end{tabular}

than settle down to a constant value. For example, the mean GAE of air temperature at $250 \mathrm{hPa}$ for the control simulation is $-0.37 \mathrm{~K}$ but its standard deviation is $0.7 \mathrm{~K}$. The nudged simulations, which have lower mean GAE, also have a corresponding lower standard deviation of GAE of $0.01-0.04 \mathrm{~K}$. This shows smaller fluctuations in the GAE of the nudged simulations and the control simulation.

Tables 1, 2 and 3 also show that the simulations that are more tightly constrained in RMSE do not necessarily result in lower GAE. This is very dependant on the variable and vertical level looked at. For example, looking at $V$ at $500 \mathrm{hPa}$ in Table 3, the hard nudging simulations which are more constrained in RMSE have a lower GAE than the control, whereas the soft nudging simulations are not noticeably improved relative to the control simulation. However, for $V$ at $250 \mathrm{hPa}$, the GAEs for all of the nudged simulations are reduced by an order of magnitude relative to the control simulation and there is very little difference between the nudged simulations. The GAE can also have the opposite trend to the RMSE. An example of this is for $T$ in Table 1, where the hard relaxation nudging has a smaller RMSE but a larger magnitude of GAE compared to the hard spectral nudging simulations. Hence, there is no nudging approach that clearly produces superior GAE results for all measures. However, the GAE for nudging simulations is comparable or lower than the control simulations for all cases, and there are only a few values that are not improved when nudging is introduced.

\subsubsection{Effect of nudging on unconstrained atmospheric fields}

In addition to constraining the nudged parameters, it is important that the nudging does not have a detrimental effect on other atmospheric processes. For this study, we examine the simulated MSLP and precipitation. We have chosen to concentrate on these fields since they can be readily compared to ERAI results and can also potentially be tested by observational data. For simplicity, in this paper we will compare the simulated results to ERAI predictions, noting that ERAI also produces an imperfect simulation of rainfall. A more detailed discussion of how nudging can effect model physics can be found in Jeuken et al. (1996). The spatial distributions of the RMSE of MSLP and precipitation are shown in Figs. 5 and 6, respectively, for the different nudging methods discussed in this paper. The results show a reduction in the RMSE in the nudged simulations (Fig. 5b-d) compared to the control simulation (Fig. 5a), illustrating that the simulated MSLP is responding favourably to the nudging. Differences between the ACCESS-simulated MSLP and ERAI are more noticeable for regions of high orography, although this may be attributable to differences in the method used to calculate MSLP under orography. The magnitude of the 
Table 3. Comparison of RMSE and GAE in $V$ measured in metres per second, for 1-year simulations excepting the first 10 days, using different nudging methods. Spectral nudging experiments use nudging applied once an hour.

\begin{tabular}{lrrrrrrr}
\hline Experiment & \multicolumn{3}{c}{ RMSE } & & \multicolumn{3}{c}{ GAE } \\
\cline { 2 - 5 } \cline { 6 - 8 } & $250 \mathrm{hPa}$ & $500 \mathrm{hPa}$ & $850 \mathrm{hPa}$ & & $250 \mathrm{hPa}$ & $500 \mathrm{hPa}$ & $850 \mathrm{hPa}$ \\
\hline Control & 18.0 & 11.7 & 7.84 & & -0.061 & 0.021 & -0.034 \\
Relaxation, soft & 1.47 & 1.09 & 1.34 & & 0.006 & 0.020 & 0.003 \\
Relaxation, hard & 0.92 & 0.70 & 1.00 & & 0.006 & 0.011 & 0.004 \\
Spectral, soft, $\lambda=0.1$ & 2.63 & 2.21 & 2.16 & & 0.006 & 0.022 & 0.001 \\
Spectral, hard, $\lambda=0.03$ & 1.16 & 0.91 & 1.14 & & 0.007 & 0.013 & 0.007 \\
Spectral, hard, $\lambda=0.1$ & 1.72 & 1.47 & 1.65 & & 0.007 & 0.014 & 0.012 \\
Spectral, hard, $\lambda=0.2$ & 3.47 & 2.96 & 2.57 & & 0.006 & 0.016 & 0.011 \\
\hline
\end{tabular}

MSLP RMSE is similar for each of the nudged simulations, although MSLP is a relatively smooth field and can be less sensitive to smaller-scale differences in the nudging.

In Fig. 6 we consider the RMSE of the monthly mean rainfall. To cover the whole seasonal cycle, the 1-year simulation including the first 10 days was used in this analysis. As the ERAI precipitation is calculated by a model with different physics to ACCESS1.3, differences in specific rainfall events are expected. Due to this and the significant spatial and temporal variability in rainfall, the monthly mean values were chosen to provide a more consistent interpretation of precipitation biases than comparing higher frequency data. This only allows us to evaluate the spatial distribution of the rainfall rather than the timing of rainfall events. Each of the nudged simulations have improved the monthly precipitation compared to the control simulation. However, Fig. 6b shows a worsening of the RMSE for the hard relaxation method over a few limited regions such as the Himalayas and Andes compared to the control simulation (Fig. 6a). This issue is also present in the soft relaxation nudging simulation (not shown) and this result is consistent with Zhang et al. (2014), who found that Newtonian relaxation could have a detrimental effect on the cloud and precipitation processes due to temperature nudging. However, Fig. $6 \mathrm{c}$ and d, using spectral nudging, have reduced this issue or even removed the problem in some locations. It is clear that leaving smaller length scales unperturbed by the spectral filter is advantageous for the model physical parametrizations at least when simulating rainfall processes, even for the relatively strong nudging case shown in Fig. 6.

\subsection{Evaluation of 1-D filter approximation}

The majority of results presented in this manuscript are for nudging simulations using the 1-D filter. To justify this choice of spectral filter method, in this section we compare the results of different configurations of the 1-D filter to those obtained using the 2-D filter. There are two ways to order the convolutions in the 1-D filter, with the zonal convolution followed by the meridional convolution (1-D filter, long-lat), or

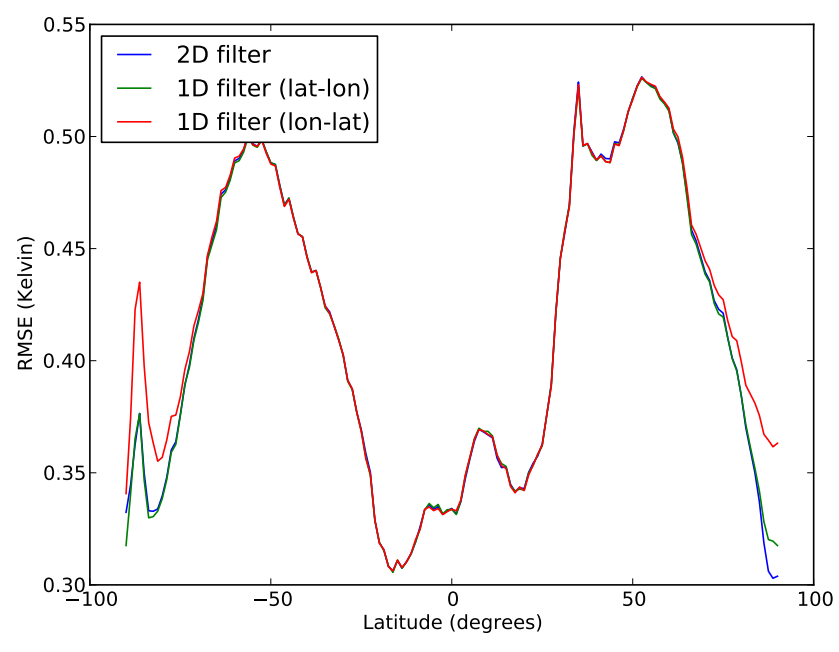

Figure 7. RMSE of air temperature at $500 \mathrm{hPa}$, of 1-D filters and 2-D filter compared to ERAI. Data was averaged temporally and zonally, for 1 year (excepting from the first 10 days) of data sampled every $6 \mathrm{~h}$. Each simulation uses the same nudging parameters, with hard nudging, using a filter length scale of $\lambda=0.1$, applied once an hour.

the meridional convolution followed by the zonal convolution (1-D filter, lat-long).

The RMSE of air temperature at $500 \mathrm{hPa}$ is very similar between the different methods of spectral nudging. Simulations using hard nudging and a filter length of $\lambda=0.1$ applied once an hour give an RMSE of $0.407 \mathrm{~K}$ for the 2-D filter and the 1-D filter lat-long, compared to $0.408 \mathrm{~K}$ for the 1-D filter long-lat, in air temperature at $500 \mathrm{hPa}$, over a 1-year simulation (excepting the first 10 days).

To more closely compare the different ordering of the 1-D convolutions, Fig. 7 shows the zonal mean RMSEs of simulations using the 1-D filters and the 2-D filter. In polar regions, there is a greater RMSE in the 1-D long-lat case compared to the 2-D filter and the 1-D lat-long. This indicates that the lat-long case is a better approximation of the 2-D filter than the long-lat. 


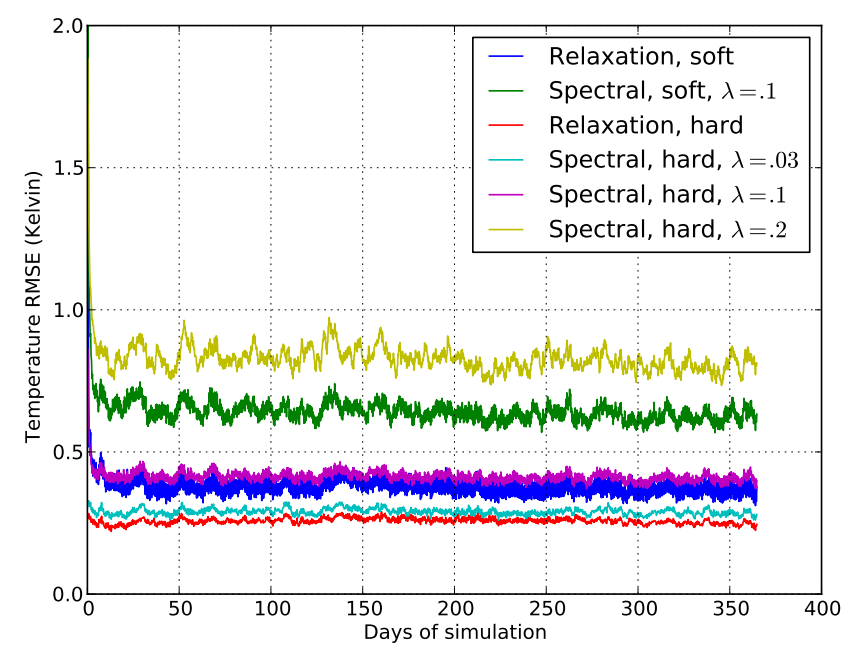

Figure 8. RMSE of temperature at $500 \mathrm{hPa}$, for a 1-year simulation. Simulations of relaxation and spectral nudging are compared, with strong or weak nudging, and several different spectral filter length scales. All of the spectral nudging simulations use the 1-D filter nudged once an hour.

The difference between the long-lat and the lat-long version of the 1-D filter occurs because the grid points near the pole are physically close together in the longitudinal direction. A small error at the pole could be spread zonally across multiple grid points. In the long-lat case, this error will remain after the initial zonal convolution. On the other hand, when the meridional convolution is applied first, the error near the poles can be reduced. This is because the values at grid boxes close to the poles have a smaller weighting in the meridional convolution, as they have a smaller area.

As the 1-D filter constrains the model to a similar extent as the 2-D filter, with much reduced computational effort, it is clearly the preferred choice. The 1-D filter with the meridional convolution applied first has better performance at the poles, so it is the optimum configuration. All simulations using spectral nudging refer to this configuration, except where specified otherwise.

\subsection{Performance of the spectral filter}

Figure 8 shows time series of RMSE of air temperature at $500 \mathrm{hPa}$ for relaxation and spectral nudging simulations, using different filter length scales and $e$-folding times. Each spectral nudging simulation uses hourly nudging as discussed in Sect. 3.4. The convergence of RMSE depends on the combination of the $e$-folding time and nudging length scale (for the spectral nudging). The model is more tightly constrained using the shorter $e$-folding time (hard nudging) and smaller nudging length scales. The spectral filter with $\lambda=0.1$ and a $1 \mathrm{~h} e$-folding time gives a RMSE similar to the relaxation nudging with a $6 \mathrm{~h} e$-folding time. The more tightly constrained simulations reach a steady state more quickly, and all the simulations shown have reached a steady RMSE within 4 days of simulation or less (not visible for the timescale of this plot).

Tables 1, 2 and 3 show the temporally and spatially averaged RMSE and GAE for each of the nudged fields $T, U$ and $V$ at 250,500 and $850 \mathrm{hPa}$ levels. The simulations shown in these tables have the same relationship in RMSE as shown in Fig. 8. In particular, for a given strength of nudging, the relaxation nudging has the smallest RMSE, the spectral nudging with $\lambda=0.03$ is closest to the relaxation nudging, and the RMSE increases for the spectral nudging as the filter length increases. This is true for each of the variables $T, U$ and $V$, at each level evaluated. In addition, the hard nudging simulations result in smaller RMSE than the equivalent set-up using soft nudging.

As seen in Sect. 3.1.1, the GAE is generally improved in comparison to the control simulation. In addition, the hard spectral nudging with the smallest filter length, $\lambda=0.03$, produces GAEs that are reasonably consistent with the relaxation nudging results. However, when comparing the different nudging methods, there is no clear pattern across levels and variables. For hard nudging, the spectral filter simulations have a smaller temperature GAE at all levels compared to the relaxation nudging, but the same is not the case when looking at the soft nudging simulations or when evaluating $U$ and $V$. Hence, there is no clear advantage of any particular nudging method when evaluating the model performance in terms of GAE.

To further show the effect of the spectral filter at different length scales, the simulation output was re-gridded to a range of coarser resolutions. Re-gridding to coarser resolutions removes the fine-scale detail in a similar way to the spectral filter, so the performance of the spectral filter should improve at coarser resolutions. This is shown in Fig. 9, which compares the RMSE at different re-gridded resolutions for different simulations.

At the highest resolutions, the relaxation nudging has a smaller RMSE, showing that it constrains the small length scales more tightly than the spectral nudging. For the spectral nudging, decreasing $\lambda$ reduces the RMSEs at all length scales (i.e. shifts the curve downward). At coarser re-gridded resolutions, the spectral nudging simulations with $\lambda=0.1$ and $\lambda=0.2$ have lower RMSE than the relaxation nudging. Hence, the spectral nudging can capture the large-scale structures of ERAI better than the relaxation nudging. The spectral nudging with $\lambda=0.5$ has a greater RMSE for all re-gridded resolutions apart from the largest, indicating that the filter is not as effective at constraining the model in this case. From this we can choose relaxation nudging or spectral nudging with a smaller or greater $\lambda$, depending on which spatial length scales we want to constrain. 


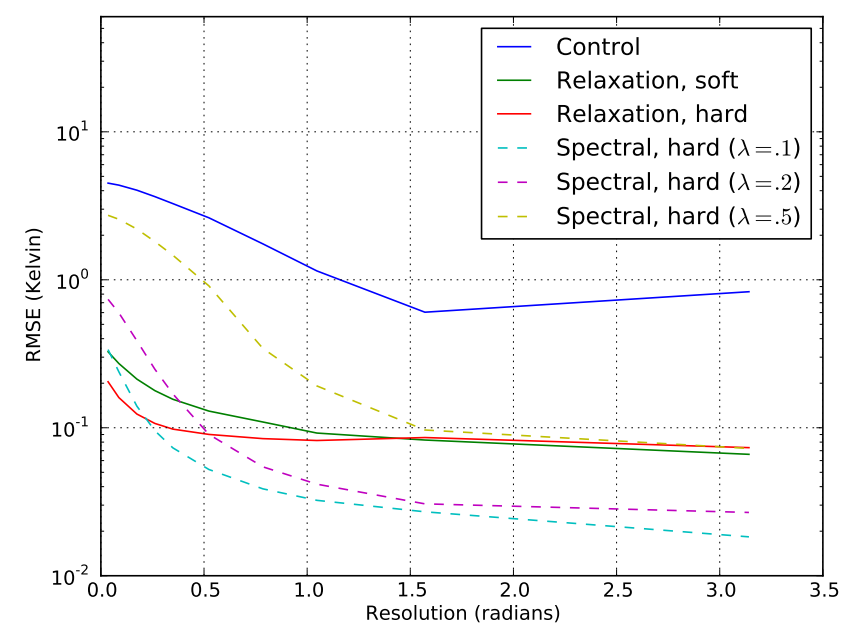

Figure 9. Plot of average RMSE of temperature at $500 \mathrm{hPa}$, at different regridded resolutions, for various simulations using nudging and a control simulation without nudging. All of the spectral nudging simulations use the 1-D filter nudged once an hour.

\subsection{Nudging period}

Figure 10 shows the temporal spectra of the $500 \mathrm{hPa}$ air temperature from simulations using different nudging configurations. Relaxation nudging is applied every time step, so the nudging period is only applicable to the spectral filter. Nudging can be applied at intervals from every time step (30 min), to the period of the host data ( $6 \mathrm{~h}$ in the case of ERAI).

All valid choices of nudging period are able to sufficiently constrain the model, given a comparable $e$-folding time. The choice of nudging, therefore, is a trade-off between computational effort and increased nudging shock, as constraining the model when nudging less frequently requires larger adjustments to the perturbed field. Nudging less frequently hence causes distortions to the temporal spectra as shown in Fig. 10. However, less frequent nudging offers a significant speedup as discussed below.

Examining Fig. 10 in more detail, it is evident that nudging with a period of $6 \mathrm{~h}$ results in spikes in the Fourier spectrum at certain frequencies. This shows that the nudging adjustment is unbalancing the atmospheric model, causing it to respond unevenly in the spectrum. When nudging every hour, these imbalances are removed. Apart from a distortion in the spectrum below half an hour (one time step), the line for spectral nudging every hour lies on top on the line for spectral nudging at every time step.

The spectra when nudging every time step is qualitatively similar to the control simulation but shifted down in magnitude. The spectral nudging at every time step has a spectrum in between the curves for the control and relaxation nudging. The spectrum for the 2-D filter is indistinguishable to the equivalent simulations using the 1-D spectral filter with the same filter length scale (2-D filter not shown).

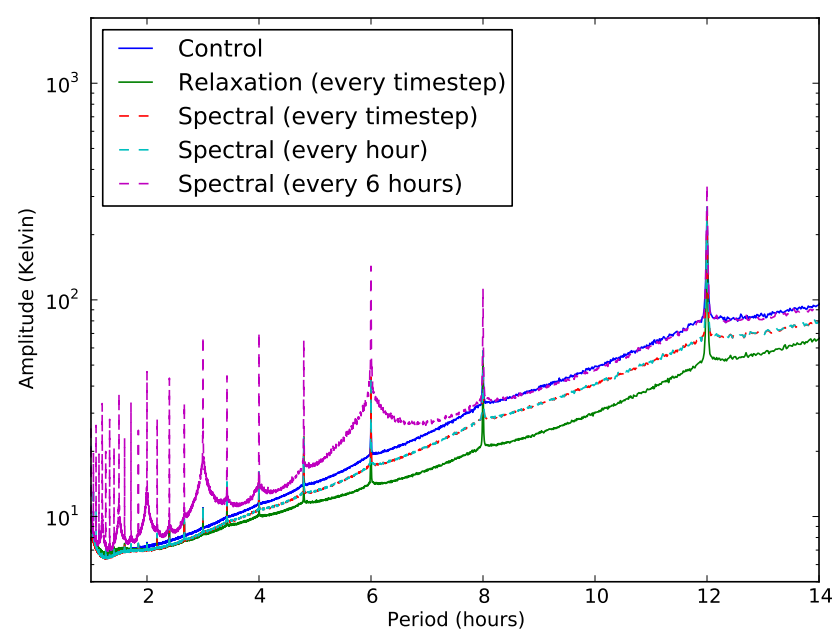

Figure 10. Temporal Fourier spectra for temperature at $500 \mathrm{hPa}$, for simulations with different nudging periods. Soft nudging was applied and the spectral nudging simulations used a filter length scale of $\lambda=0.1$.

Considering the speed benefits of different nudging frequencies, the 1-D spectral filter nudged every $6 \mathrm{~h}$ adds $3.3 \%$ to the run time (the same as Newtonian relaxation). When the period is decreased to $1 \mathrm{~h}$ or $30 \mathrm{~min}$ this increases the run time by 6.7 and $12 \%$, respectively. The 2-D spectral filter in comparison adds $33 \%$ when nudged every $6 \mathrm{~h}$, increasing to 190 and $376 \%$, which is not viable for most uses.

Nudging at hourly intervals can be used as a compromise between speed of computation and reducing the distortions in the spectra, and is the standard period of nudging used for spectral nudging in this paper.

\section{Conclusions}

This paper has introduced the use of spectral nudging in the UM and ACCESS. This is achieved through a novel convolution method, first described by Thatcher and McGregor (2009), but generalized in this paper for use with latitudelongitude grids as used by the ACCESS atmospheric model. Analysis of the different configurations of nudging shows that the nudging schemes effectively constrain the nudged fields to follow the host model (ERAI). We have surveyed the spectral filter across a range of filter length scales. The spectral nudging scheme approaches the Newtonian relaxation nudging when small length scales are nudged, but allows the flexibility to nudge only large spatial structures when the filter length scale is increased.

Our results show that simulation errors in air temperature are greater near the surface for all nudging methods, which is expected due to the different representation of land-surface parametrizations. Although our objective was to compare the Newtonian relaxation with the spectral filter in ACCESS, we note that differences occur where there is a mismatch in 
the orographic height between the ACCESS simulation and ERAI, suggesting a problem with the vertical interpolation to the ACCESS grid used by the nudging. We intend to address this problem in future work.

We have also considered the implications of nudging on MSLP and precipitation, which are not directly perturbed by the nudging. MSLP is a reasonably smoothly varying field and is well constrained by the nudging in all simulations to agree with ERA-Interim. There are some differences under high orography, although this may be more related to the method used for calculating MSLP under orography rather than the nudging method. The nudged simulation improved the monthly mean rainfall compared to the control simulation. Furthermore, the spectral nudging simulations predicted rainfall that was in closer agreement with ERAI than the relaxation nudging simulations. This provides an example of where the spectral filter can have an advantage over the Newtonian relaxation approach, particularly for physical processes that are sensitive to the local behaviour of the atmosphere.

The 1-D spectral filter is shown to perform as well as the 2-D filter, while producing a speedup of 10-30 times. This is achieved by the approximation of separating the 2-D convolution into 1-D convolutions and by using symmetries of the model grid to reduce communication between processors. We also identified that, due to the geometry of our grid, the order of convolutions in the 1-D filter was important. To reduce error in the approximation, the meridional convolution is applied first.

Nudging with different frequencies was also investigated, showing that nudging every $6 \mathrm{~h}$ is still able to constrain the model, but introduces distortions to the spectra. Nudging once an hour produces a speedup in comparison to nudging every time step, while introducing minimal distortions, so it was used for the majority of simulations.

The approach used to implement the 2-D and 1-D spectral filters is applicable to many other models. The 2-D convolution method can be implemented on any grid, though it suffers from being computationally expensive. The 1-D filter can be applied to irregular or more complex grids, but would require modification to separate the 2-D Gaussian function using an approximation that is appropriate for the particular grid.

Future work on spectral nudging in ACCESS will involve generalizing the spectral nudging to limited area and stretched grid configurations. Another potential approach to gaining a speedup in the convolution-based spectral filter is to compute the convolutions over a small neighbourhood, rather than the whole globe, ignoring areas where the Gaussian function has values close to 0 . The ability to extend the convolution-based spectral filter within the ACCESS/UM framework and in other modelling systems is an advantage of this approach.

\section{Code availability}

Due to intellectual property right restrictions, CSIRO cannot publish the full source code for ACCESS or the UM. The Met Office Unified Model (UM) with the spectral nudging source code and configuration described in this paper can be obtained under an end-user license agreement (EULA) from CSIRO for educational and non-commercial research use for specific projects. To request a EULA for the modified UM, and/or to obtain the ACCESS1.3 model configuration used in this paper, please contact Tony Hirst (tony.hirst@csiro.au).

Acknowledgements. Thanks to Peter Dobrohotoff, John McGregor and Tony Hirst for their feedback in the preparation of the manuscript and the anonymous reviewers for suggested revisions to improve the manuscript. This research was undertaken with the assistance of resources from the National Computational Infrastructure (NCI), which is supported by the Australian Government. This work included funding by the Australian Government through the Australian Climate Change Science Programme. ERA-Interim data, from the European Centre for Medium-Range Weather Forecasts (ECMWF) was used in this research. The UM was made available to CSIRO under the consortium agreement Met Office's Unified Model Earth System Modelling software (Met Office Ref. L1587).

Edited by: G. Mann

\section{References}

Bi, D., Dix, M., Marsland, S., O'Farrell, S., Rashid, H., Uotila, P., Hirst, A., Kowalczyk, E., Golebiewski, M., Sullivan, A., Yan, H., Hannah, N., Franklin, C., Sun, Z., Vohralik, P., Watterson, I., Zhou, X., Fiedler, R., Collier, M., Ma, Y., Noonan, J., Stevens, L., Uhe, P., Zhu, H., Griffies, S., Hill, R., Harris, C., and Puri, K.: The ACCESS coupled model: description, control climate and evaluation, Aust. Met. Oceanogr. J., 63, 41-64, 2013.

Davies, T., Cullen, M. J. P., Malcolm, A. J., Mawson, M. H., Staniforth, A., White, A. A., and Wood, N.: A new dynamical core for the Met Office's global and regional modelling of the atmosphere, Q. J. Roy. Meteorol. Soc., 131, 1759-1782, doi:10.1256/qj.04.101, 2005.

Dee, D. P., Uppala, S. M., Simmons, A. J., Berrisford, P., Poli, P., Kobayashi, S., Andrae, U., Balmaseda, M. A., Balsamo, G., Bauer, P., Bechtold, P., Beljaars, A. C. M., van de Berg, L., Bidlot, J., Bormann, N., Delsol, C., Dragani, R., Fuentes, M., Geer, A. J., Haimberger, L., Healy, S. B., Hersbach, H., Hólm, E. V., Isaksen, L., Kållberg, P., Köhler, M., Matricardi, M., McNally, A. P., Monge-Sanz, B. M., Morcrette, J.-J., Park, B.K., Peubey, C., de Rosnay, P., Tavolato, C., Thépaut, J.-N., and Vitart, F.: The ERA-Interim reanalysis: configuration and performance of the data assimilation system, Q. J. Roy. Meteorol. Soc., 137, 553-597, doi:10.1002/qj.828, 2011.

Denis, B., Coté, J., and Laprise, R.: Spectral decomposition of twodimensional atmospheric fields on limited-area domains using the discrete cosine transform (DCT), Mon. Weather Rev., 130, 1812-1829, 2002. 
Dix, M., Vohralik, P., Bi, D., Rashid, H., Marsland, S., O’Farrell, S., Uotila, P., Hirst, T., Kowalczyk, E., Sullivan, A., Yan, H., Franklin, C., Sun, Z., Watterson, I., Collier, M., Noonan, J., Rotstayn, L., Stevens, L., Uhe, P., and Puri, K.: The ACCESS couple model: documentation of core CMIP5 simulations and initial results, Aust. Met. Oceanogr. J., 63, 83-99, 2013.

Jeuken, A., Siegmund, P., Heijboer, L., Feichter, J., and Bengtsson, L.: On the potential of assimilating meteorological analyses in a global climate model for the purpose of model validation, J. Geophys. Res.-Atmos., 101, 16939-16950, 1996.

Kanamaru, H. and Kanamitsu, M.: Scale-selective bias correction in a downscaling of global analysis using a regional model, Mon. Weather Rev., 135, 334-350, 2007.

Kida, H., Koide, T., Sasaki, H., and Chiba, M.: A new approach for coupling a limited area model to a GCM for regional climate simulations, J. Meteorol. Soc. Jpn., 69, 723-728, 1991.

Koffi, E. N., Rayner, P. J., Scholze, M., and Beer, C.: Atmospheric constraints on gross primary productivity and net ecosystem productivity: results from a carbon-cycle data assimilation system, Global Biogeochem. Cy., 26, 1-15, doi:10.1029/2010GB003900, 2012.

Kowalczyk, E., Stevens, L., Law, R., Dix, M., Wang, Y., Harman, I., Haynes, K., Srbinovsky, J., Pak, B., and Ziehn, T.: The land surface model component of ACCESS: description and impact on the simulated surface climatology, Aust. Met. Oceanogr. J., 63, 65-82, 2013.

Liu, P., Tsimpidi, A. P., Hu, Y., Stone, B., Russell, A. G., and Nenes, A.: Differences between downscaling with spectral and grid nudging using WRF, Atmos. Chem. Phys., 12, 3601-3610, doi:10.5194/acp-12-3601-2012, 2012.

Puri, K., Dietachmayer, G., Steinle, P., Dix, M., Rikus, L., Logan, L., Naughton, M., Tingwell, C., Xiao, Y., Barras, V., Bermous, I., Bowen, R., Deschamps, L., Franklin, C., Fraser, J., Glowacki, T., Harris, B., Lee, J., Le, T., Roff, G., Sulaiman, A., Sims, H., Sun, X., Sun, Z., Zhu, H., Chattopadhyay, M., and Engel, C.: Implementation of the initial ACCESS numerical weather prediction system, Aust. Met. Oceanogr. J., 63, 265-284, 2013.

Taylor, K., Stouffer, R. L., and Meehl, G. A.: An Overview of CMIP5 and the experiment design, B. Am. Meteorol. Soc., 93, 485-498, doi:10.1175/BAMS-D-11-00094.1, 2012.
Telford, P. J., Braesicke, P., Morgenstern, O., and Pyle, J. A.: Technical Note: Description and assessment of a nudged version of the new dynamics Unified Model, Atmos. Chem. Phys., 8, 17011712, doi:10.5194/acp-8-1701-2008, 2008.

Thatcher, M. and McGregor, J. L.: Using a scale-selective filter for dynamical downscaling with the conformal cubic atmospheric model, Mon. Weather Rev., 137, 1742-1752, doi:10.1175/2008MWR2599.1, 2009.

The HadGEM2 Development Team: Martin, G. M., Bellouin, N., Collins, W. J., Culverwell, I. D., Halloran, P. R., Hardiman, S. C., Hinton, T. J., Jones, C. D., McDonald, R. E., McLaren, A. J., O’Connor, F. M., Roberts, M. J., Rodriguez, J. M., Woodward, S., Best, M. J., Brooks, M. E., Brown, A. R., Butchart, N., Dearden, C., Derbyshire, S. H., Dharssi, I., Doutriaux-Boucher, M., Edwards, J. M., Falloon, P. D., Gedney, N., Gray, L. J., Hewitt, H. T., Hobson, M., Huddleston, M. R., Hughes, J., Ineson, S., Ingram, W. J., James, P. M., Johns, T. C., Johnson, C. E., Jones, A., Jones, C. P., Joshi, M. M., Keen, A. B., Liddicoat, S., Lock, A. P., Maidens, A. V., Manners, J. C., Milton, S. F., Rae, J. G. L., Ridley, J. K., Sellar, A., Senior, C. A., Totterdell, I. J., Verhoef, A., Vidale, P. L., and Wiltshire, A.: The HadGEM2 family of Met Office Unified Model climate configurations, Geosci. Model Dev., 4, 723-757, doi:10.5194/gmd-4-723-2011, 2011.

von Storch, H., Langenberg, H., and Feser, F.: A spectral nudging technique for dynamical downscaling purposes, Mon. Weather Rev., 128, 3664-3673, 2000.

Waldron, K., Paegle, J., and Horel, J.: Sensitivity of a spectrally filtered and nudged limited-area model to outer model options, Mon. Weather Rev., 124, 529-547, 1996.

Wang, Y., Leung, L. R., McGregor, J. L., Lee, D.-K., Wang, W.-C., Ding, Y., and Kimura, F.: Regional climate modelling: progress, challenges and prospects, J. Meteorol. Soc. Jpn., 82, 1599-1628, 2004.

Zhang, K., Wan, H., Liu, X., Ghan, S. J., Kooperman, G. J., Ma, P.-L., Rasch, P. J., Neubauer, D., and Lohmann, U.: Technical Note: On the use of nudging for aerosol-climate model intercomparison studies, Atmos. Chem. Phys., 14, 8631-8645, doi:10.5194/acp-14-8631-2014, 2014. 